\section{Omega-3 fatty acids are a potential therapy for patients with sickle cell disease}

\section{Ahmed Daak, Adrian Rabinowicz and Kebreab Ghebremeskel}

We have read with interest the excellent review on sickle cell disease (SCD) recently published in Nature Reviews Disease Primers (Sickle cell disease. Nat. Rev. Dis. Primers 4, 18010 (2018)) $)^{1}$. The Primer succinctly highlights the recent advances in the molecular pathology of SCD, medical management options and future challenges. However, the article did not underscore the crucial role of abnormality in the membrane lipids of blood cells in the pathogenesis of the disease, as well as the therapeutic potential of long-chain omega- 3 fatty acids demonstrated in pilot and randomized studies ${ }^{2-6}$.

The authors stated, "HbS polymerization directly or indirectly alters the typical lipid bilayer and proteins of the erythrocyte membrane"; however, they did not mention that the plasma membrane of red blood cells, white blood cells and platelets of patients with SCD has an abnormal fatty acid composition, characterized by decreased levels of docosahexaenoic acid (DHA, 22:6 $\omega 3$ ) and eicosapentaenoic acid (EPA, 20:5 $\omega 3$ ), and a concomitant increase in arachidonic acid

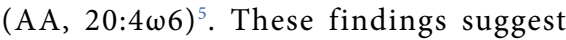
that membrane lipid perturbation and its manifestations, including the exposure of phosphatidyl serine on the exterior of red blood cell membranes and the propensity of red blood cells, white blood cells and platelets to adhere and aggregate, is fundamental to SCD pathology.

Tomer et $\mathrm{al}^{2}$ reported a lower frequency of pain episodes in patients with SCD who were treated with omega- 3 fatty acids than in patients treated with placebo (olive oil). Subsequently, Daak et al. ${ }^{5}$ conducted a large $(n=140)$ single-centre randomized, doubleblind, placebo-controlled trial with omega-3 fatty acids in patients with homozygous sickle cell anaemia (HbSS). The annualized vaso-occlusive crisis (VOC) rate and the rate and length of hospitalizations for VOC were significantly lower in the omega- 3 group than in patients who received placebo capsules. We have recently concluded a phase II multicentre randomized, double-blind, placebocontrolled, parallel-group, dose-finding trial (SCOT) in the United States. Consistent with previous findings, our data reveals a $>53 \%$ reduction in clinical VOCs in patients with SCD treated with DHA. A detailed protocol of the SCOT trial is provided on the ClinicalTrials.gov website.

These clinical studies provide evidence that omega- 3 fatty acids are effective and safe treatment options for patients with SCD. The specific mechanisms through which these fatty acids ameliorate VOCs are yet to be elucidated. Nevertheless, omega-3 fatty acids and their active metabolites have wellsubstantiated pleiotropic biological actions anti-inflammatory, inflammation resolving, anti-adhesion, anti-aggregation, vasodilatory and antioxidant ${ }^{7-10}$. In addition, there is evidence that abnormality of membrane fatty acids, rheological abnormalities of red blood cells, inflammation and haemolysis in patients with SCD are ameliorated by treatment with omega-3 fatty acids ${ }^{10-13}$.

After hydroxycarbamide received approval $>20$ years ago, only one drug, L-glutamine, has been recently approved for SCD, whereas most drug development programmes have failed to live up to their earlier promise. This is to be expected because of the complex nature of SCD pathophysiology. Hence, a multimodal therapy or a single drug with multiple mechanisms of action may be necessary to achieve clinical effectiveness $^{14,15}$. The multipronged biological and pleiotropic effects of omega-3 fatty acids could make them a new therapeutic option for patients with SCD.

Ahmed Daak ${ }^{1,2 *}$, Adrian Rabinowicz ${ }^{1,2}$ and Kebreab Ghebremeskel ${ }^{3}$

Center of Molecular Biology and Biotechnology (CMBB), Florida Atlantic University, Jupiter, FL, USA.
2Sancilio Pharmaceuticals Company, Riviera Beach, FL, USA.

${ }^{3}$ Lipidomics and Nutrition Research Centre, London Metropolitan University, London, UK.

*e-mail:adaak@fau.edu https://doi.org/10.1038/s41572-018-0012-9

1. Kato, G. J. et al. Sickle cell disease. Nat. Rev. Dis. Primers 4, 18010 (2018)

2. Tomer, A. et al. Reduction of pain episodes and prothrombotic activity in sickle cell disease by dietary $\mathrm{n}-3$ fatty acids. Thromb. Haemost. $\mathbf{8 5}$, 966-974 (2001).

3. Daak, A. A. et al. Effect of omega-3 (n-3) fatty acid supplementation in patients with sickle cell anemia: randomized, double-blind, placebo-controlled trial. Am. J. Clin. Nutr. 97, 37-44 (2012).

4. Sins, J. W. R. et al. Pharmacotherapeutical strategies in the prevention of acute, vaso-occlusive pain in sickle cell disease: a systematic review. Blood Adv. 1, 1598-1616 (2017).

5. Ren, H. et al. Blood mononuclear cells and platelets have abnormal fatty acid composition in homozygous sickle cell disease. Ann. Hematol. 84, 578-583 (2005).

6. Kuypers, F. A. Membrane lipid alterations in hemoglobinopathies. Hematology Am. Soc. Hematol. Educ. Program 2007, 68-73 (2007).

7. Calder, P. C. Marine omega-3 fatty acids and inflammatory processes: effects, mechanisms and clinical relevance. Biochim. Biophys. Acta 1851, 469-484 (2015).

8. Buckley, C. D., Gilroy, D. W. ¿ Serhan, C. N. Proresolving lipid mediators and mechanisms in the resolution of acute inflammation. Immunity 40, 315-327 (2014).

9. Mozaffarian, D. \& Wu, J. H. Omega-3 fatty acids and cardiovascular disease: effects on risk factors, molecular pathways, and clinical events. J. Am. Coll. Cardiol. 58, 2047-2067 (2011)

10. Daak, A. A. et al. Omega 3 (n-3) fatty acids down-regulate nuclear factor-kappa B (NF-kappaB) gene and blood cell adhesion molecule expression in patients with homozygous sickle cell disease. Blood Cells Mol. Dis. 55, 48-55 (2015).

11. Daak, A. A et al. Docosahexaenoic and eicosapentaenoic acid supplementation does not exacerbate oxidative stress or intravascular haemolysis in homozygous sickle cell patients. Prostaglandins Leukot Essent. Fatty Acids $\mathbf{8 9}$, 305-311 (2013).

12. Kalish, B. T. et al. Dietary omega-3 fatty acids protect against vasculopathy in a transgenic mouse model of sickle cell disease. Haematologica 100, 870-880 (2015).

13. Wandersee, N. J. et al. Dietary supplementation with docosahexanoic acid (DHA) increases red blood cell membrane flexibility in mice with sickle cell disease. Blood Cells Mol. Dis. 54, 183-188 (2015).

14. Jagadeeswaran, R. \& Rivers, A. Evolving treatment paradigms in sickle cell disease. Hematology Am. Soc. Hematol. Educ. Program 2017, 440-446 (2017).

15. Hebbel, R. P., Vercellotti, G. \& Nath, K. A. A systems biology consideration of the vasculopathy of sickle cell anemia: the need for multi-modality chemoprophylaxsis. Cardiovasc. Hematol. Disord. Drug Targets 9, 271-292 (2009).

\section{Competing interests}

A.D. and A.R. are employees of Sancilio Pharmaceuticals Inc (SCI). K.G. declares no competing interests.

\section{Publisher's note}

Springer Nature remains neutral with regard to jurisdictional claims in published maps and institutional affiliations.

RELATED LINKS

SCOT trial: https://clinicaltrials.gov/ct2/show/NCT02973360 